\title{
Effect of CyberKnife stereotactic body radiation therapy for hepatocellular carcinoma on hepatic toxicity
}

Ping Liang, 1 * Cheng Huang, ${ }^{2} *$ Shi-Xiong Liang, ${ }^{2,3}$ Ye-Fei Li, ${ }^{3}$ Shang-Xiao Huang, ${ }^{2}$ Zu-Ping Lian,' Jian-Min Liu,' Yang Tang,' Hai-Jie Lu ${ }^{4}$

'Department of Radiation Oncology, Ruikang Hospital Affiliated to Guangxi University of Chinese Medicine, ${ }^{2}$ Department of Radiation Oncology, Cancer Hospital, Guangxi Medical University, Nanning, ${ }^{3}$ Department of Radiation Oncology, Shanghai Pulmonary Hospital, Tongji University School of Medicine, Shanghai, ${ }^{4}$ Department of Radiation Oncology, Zhongshan Hospital of Xiamen University, Xiamen, Fujian, People's Republic of China

*These authors contributed equally to this work

Correspondence: Shi-Xiong Liang Department of Radiation Oncology, Shanghai Pulmonary Hospital, Tongji University School of Medicine, 507,

Zhengmin Road, Shanghai, 200433,

People's Republic of China

Tel +86 I39 I77| 6605

Fax +86 2I 65II 5006

Email shixliang@vip.sina.com

Hai-Jie Lu

Department of Radiation Oncology,

Zhongshan Hospital of Xiamen

University, 209 Hubin South Road,

Xiamen, Fujian, 361004, People's Republic of China

Tel +86 I $33659 \mid 7208$

Fax +86 5922212328

Email luhaijie1971@hotmail.com
This article was published in the following Dove Press journal:

OncoTargets and Therapy

18 November 2016

Number of times this article has been viewed

Objective: To evaluate the safety of CyberKnife stereotactic body radiation therapy (SBRT) for hepatocellular carcinoma (HCC) patients and identify the treatment-related risk factors of hepatic toxicity.

Materials and methods: One hundred and four HCC patients treated with CyberKnife SBRT were included in this study between August 2009 and December 2012. The average dose of prescribed radiation was $42.81 \pm 4.78$ Gy (28-55 Gy) with the average fraction size of 8-16 Gy to the planning target volume. The average fractions were $3.31 \pm 0.81$ (2-6 fractions). Response rates were determined, and the Child-Pugh (CP) score and class following CyberKnife SBRT were obtained to evaluate hepatic toxicity.

Results: Seventeen patients experienced progression in CP class and 24 patients experienced CTCAE V. 4.0 grade 2-3 hepatic toxicity during the five-month follow-up period, while no patient experienced grade 4 liver toxicity. Multivariate analysis indicated that only $\mathrm{V}_{25}$ was an independent factor in grade $2-3$ hepatic toxicity $(P=0.029,<0.05)$. Radiation-induced hepatic toxicity (RIHT), defined as an increase of at least two points within three months following CyberKnife SBRT, occurred in 13 of the 104 patients (13/104, 12.5\%), and only the normal liver tissue was found to be associated with RIHT $(P=0.008,<0.05)$.

Conclusion: CyberKnife SBRT is a feasible and safe treatment for HCC with regard to hepatic toxicity, while $\mathrm{V}_{25}$ and normal liver volume may be an independent factor of grade $2-3$ hepatic toxicity and RIHT, respectively.

Keywords: hepatocellular carcinoma, hepatic toxicity, CyberKnife stereotactic body radiation therapy (SBRT)

\section{Introduction}

Hepatocellular carcinoma (HCC) is one of the most common malignant diseases worldwide, and the incidence of HCC is rising in many countries. ${ }^{1-3}$ Early-stage HCC patients with a preserved liver function of Child-Pugh (CP) A or B, and up to three nodules $<3 \mathrm{~cm}$ in size, can be effectively treated by resection, liver transplantation, or percutaneous radiofrequency ablation. The five-year survival rates range between $50 \%$ and $75 \% .{ }^{4}$ However, the majority of $\mathrm{HCC}$ patients are ineligible for surgery due to the size or location of the tumor, or poor liver function as a result of liver cirrhosis. Several other modalities including percutaneous ethanol injection, transarterial chemoembolization (TACE), and sorafenib (an orally active multikinase inhibitor approved in the European Union for the treatment of HCC) have been used as palliative treatments, but the optimal treatment remains elusive. ${ }^{5-7}$ 
CyberKnife is a new frameless robotic stereotactic body radiation therapy (SBRT) means of delivering radiotherapy, intended to target treatment more accurately and rapidly than conformal radiotherapy or intensity-modulated radiotherapy (IMRT) ${ }^{8,9}$ It has been used in the treatment of benign tumors, malignant tumors, and several other medical conditions. ${ }^{10,11}$ CyberKnife SBRT has been proven to be a safe and effective noninvasive treatment for $\mathrm{HCC}$ in patients ineligible for local ablation or surgical resection; $;{ }^{12}$ however, immediate liver injury following CyberKnife treatment still needs to be determined. Radiation-induced hepatic toxicity (RIHT), defined as an increase in at least two points within 3 months, is considered a significant dose-limiting factor in HCC radiotherapy. This underlines the importance of identifying a parameter that can predict and prevent severe hepatic toxicity.

Recently, several reports investigating the parameters capable of predicting RIHT have been published. ${ }^{13-15}$ To our knowledge, there have been only a few case series evaluating RIHT following CyberKnife treatment in HCC patients. Therefore, we recruited HCC patients who were treated with CyberKnife SBRT in order to analyze the changes in the CP score and class, as well as to evaluate hepatic toxicity and RIHT. This report focused on evaluating the safety of hepatic toxicity and identifying the treatment-related risk factors in HCC treated with CyberKnife SBRT.

\section{Materials and methods}

\section{Patient information and CyberKnife SBRT details}

Between August 2009 and December 2012, a total of 123 patients with $\mathrm{HCC}$ were treated with CyberKnife SBRT in Ruikang Hospital Affiliated to the Guangxi University of Chinese Medicine. The patient inclusion criteria were as follows: 1) aged between 20 and 80 years; 2) definitive diagnosis of HCC based on the computed tomography (CT) scans and alpha fetal protein level; ${ }^{16}$ 3) CP class A or B; 4) no previous history of TACE, conventional surgery, or chemotherapy; 5) Eastern Cooperative Oncology Group (ECOG) score 0-2; and 6) no intrahepatic disease progression within three months after the completion of CyberKnife SBRT. Exclusion criteria included: 1) CP class $C$, severe portal hypertension, past history of gastric and esophageal variceal bleeding, serious hypersplenism, or refractory ascites; 2) complications with other types of viral hepatitis other than hepatitis B; and 3) pathologically confirmed cholangiocarcinoma, mixed liver cell cancer, surgical margin positive tumor, or metastatic liver tumor. The advantages and disadvantages of CyberKnife SBRT were explained to the patients and written informed consent was obtained from all patients prior to the treatment. The study was approved by the institutional ethics committee of Ruikang Hospital Affiliated to the Guangxi University of Chinese Medicine.

A total of 19 patients out of the 123 HCC patients treated with CyberKnife SBRT were excluded from this study due to the fact that they received TACE, chemotherapy, or second course radiotherapy. The median age of the included 104 patients was 55, and males were predominant. Tumors were mostly tumor, node, metastasis (TNM) III (83/104, $79.8 \%)$, ECOG $2(75 / 104,72.1 \%)$, without portal vein tumor thrombus (87/104, 83.7\%), and CP A (94/104, 90.4\%). The characteristics of the $104 \mathrm{HCC}$ patients are indicated in Table 1.

SBRT was performed using the CyberKnife system. To obtain radiographic landmarks, we percutaneously implanted gold seeds under ultrasonographic guidance in the liver parenchyma near the target tumors. On the following day, the patients were vacuum immobilized in the supine position and computed tomographic (CT) images were taken to locate the tumors. The treatment plan was designed according to TPS (V. 3.4.2) and the MultiPlan ${ }^{\mathrm{TM}}$ System, and breath-holding

Table I Patient characteristics

\begin{tabular}{|c|c|c|}
\hline Variables & No of patients & $\%$ \\
\hline \multicolumn{3}{|l|}{ Gender } \\
\hline Male & 92 & 88.5 \\
\hline Female & 12 & 11.5 \\
\hline \multicolumn{3}{|c|}{ Age (years) } \\
\hline$<55$ & 51 & 49.0 \\
\hline$\geq 55$ & 53 & 51.0 \\
\hline \multicolumn{3}{|l|}{ ECOG } \\
\hline $0-1$ & 29 & 27.9 \\
\hline 2 & 75 & 72.1 \\
\hline \multicolumn{3}{|l|}{ Diagnosis } \\
\hline Clinical & 51 & 49.0 \\
\hline Pathological & 53 & 51.0 \\
\hline \multicolumn{3}{|l|}{ TNM stage } \\
\hline I & 2 & 1.9 \\
\hline II & 11 & 10.6 \\
\hline III & 83 & 79.8 \\
\hline IV & 8 & 7.7 \\
\hline \multicolumn{3}{|c|}{ Portal vein tumor thrombus } \\
\hline Yes & 17 & 16.3 \\
\hline No & 87 & 83.7 \\
\hline \multicolumn{3}{|l|}{ HBV } \\
\hline+ & 100 & 96.2 \\
\hline- & 4 & 3.8 \\
\hline \multicolumn{3}{|c|}{ Child-Pugh class } \\
\hline A & 94 & 90.4 \\
\hline B & 10 & 9.6 \\
\hline
\end{tabular}

Abbreviations: ECOG, Eastern Cooperative Oncology Group; HBV, hepatitis B virus; TNM, tumor, node, metastasis. 
techniques were applied during the CyberKnife SBRT process. The dose-volumetric parameters were calculated on the basis of dose-volume histograms (DVHs). Gross tumor volume (GTV) was defined as the contrast-enhanced tumor volume on $\mathrm{CT}$ scans or magnetic resonance imaging, and the mean GTV was determined to be $213.54 \pm 254.98 \mathrm{~cm}^{3}$ (2.53-1,347.48 $\mathrm{cm}^{3}$, median $\left.100.48 \mathrm{~cm}^{3}\right)$. Normal liver volume was calculated as total liver volume minus GTV, and the mean normal liver volume was $1,214.28 \pm 311.54 \mathrm{~cm}^{3}$ (median 1,165.26 $\mathrm{cm}^{3}$ ). The planning target volume (PTV) was generated by adding $3-5 \mathrm{~mm}$ to the GTV and $60 \%-80 \%$ of the maximum dose was defined as the prescribed dose. The average prescribed radiation dose was $42.81 \mathrm{~Gy} \pm 4.78 \mathrm{~Gy}$ (28-55 Gy, median $45 \mathrm{~Gy}$ ) with the average fraction size of $8-16$ Gy to the PTV. The average fractions were $3.31 \pm 0.81$ (2-6 fractions, median 3 fractions).

\section{Evaluation of hepatic toxicity}

During the course of the CyberKnife SBRT, all patients were visited weekly by a physician to evaluate their condition. After completion of the CyberKnife SBRT, all patients were followed-up monthly. At every visit, physical examinations and blood tests were performed to assess hepatic toxicity. Levels of aspartate transaminase, alanine transaminase, alkaline phosphatase, serum albumin and total bilirubin, and prothrombin time (PT) were examined. Ascites and hepatic encephalopathy were also evaluated. The $\mathrm{CP}$ score, which is calculated on the basis of the serum bilirubin and albumin levels, PT, and the presence and degree of ascites, or encephalopathy, is an assessment of the severity of hepatic function. ${ }^{17}$ Thus, an increase in $\mathrm{CP}$ score reflects the deterioration of hepatic function. All toxicities were assessed by common terminology criteria for adverse events (CTCAE V. 4.0) grade toxicity.

RIHT was defined as an increase of at least two points in the CP score within 3 months following the completion of CyberKnife SBRT. The effects of the treatment on HCC were evaluated via $\mathrm{CT}$ scans every $1-2$ months after completion of the treatment.

\section{Statistical analysis}

All data are expressed as median or mean and standard deviation. Comparison of the differences in the univariables between groups was performed using a chi-square test or independent $t$-test and comparison of the mean values was performed using the $t$-test or rank sum test. Cumulative survival rates were estimated using the Kaplan-Meier method. Logistic regression analysis was used for multivariate analysis. The receiver operating characteristic (ROC) curve was used to estimate the significant dosimetric parameters. Statistical analyses were performed using SPSS V. 16.0 (SPSS Inc., Chicago, IL, USA), and $P<0.05$ was considered statistically significant.

\section{Results}

\section{Tumor response and survival time}

Grade 1 or 2 non-hematological toxicities including anorexia and nausea were found in 31 cases $(29.8 \%)$. Grade 1 toxicities occurred in 20 cases, while grade 2 toxicities occurred in 11 patients. Neither the grade 1 nor grade 2 hematological toxicities were considered severe. No grade 3 or 4 nonhematological toxicities were reported, and there were no treatment-related deaths in the group.

Tumor response was determined based on the change in the maximum tumor size on serial CT scans 1-2 months following the completion of the treatment. A response was achieved in 88 patients $(80.8 \%)$ with CR in 5 patients, partial response in 79 patients, and stable disease in 9 patients. The maximal follow-up was 11-22 weeks after the start of SBRT. The median survival time of all patients was 19 months and the 1- and 2-year survival rates were $62.2 \%$ and $44.2 \%$, respectively.

\section{Evaluation of the hepatic toxicity}

One to two weeks following the treatment of CyberKnife SBRT, none of the patients had CTCAE V. 4.0 grade 2-3 hepatic toxicity. Three to ten weeks after treatment, grade $2-3$ hepatic toxicity occurred in nine patients $(9 / 104,8.7 \%)$, and 11-22 weeks after treatment, seven patients $(7 / 104,6.7 \%)$ experienced grade 2-3 hepatic toxicity. A total of 24 (24/104, $23.1 \%$ ) patients out of 104 HCC patients indicated grade 2-3 hepatic toxicity, with grade 2 recorded in 16 cases and grade 3 in eight cases, and no patients experienced grade 4 hepatic toxicity.

The CP score indicated insignificant change 1-2 weeks following CyberKnife SBRT ( $P>0.05)$, but increased significantly at 3-10 weeks and 11-22 weeks after treatment $(P<0.05$; Table 2, Figure 1). The CP score increased in

Table 2 Changes in Child-Pugh score following CyberKnife stereotactic body radiation therapy

\begin{tabular}{lllll}
\hline $\begin{array}{l}\text { Time after } \\
\text { treatment (weeks) }\end{array}$ & $\begin{array}{l}\text { O point } \\
\text { n (\%) }\end{array}$ & $\begin{array}{l}\text { I point } \\
\text { n (\%) }\end{array}$ & $\begin{array}{l}\text { 2 points } \\
\text { n (\%) }\end{array}$ & $\begin{array}{l}\text { 3 points } \\
\text { n (\%) }\end{array}$ \\
\hline $\mathrm{I}-2$ & $90(86.5)$ & $\mathrm{I} 4(13.5)$ & $0(0.00)$ & $0(0.00)$ \\
$3-10$ & $70(67.3)$ & $25(24.0)$ & $6(5.8)$ & $3(2.9)$ \\
$\mathrm{II}-22$ & $86(82.7)$ & $\mathrm{II}(10.6)$ & $3(2.9)$ & $4(3.8)$ \\
\hline
\end{tabular}




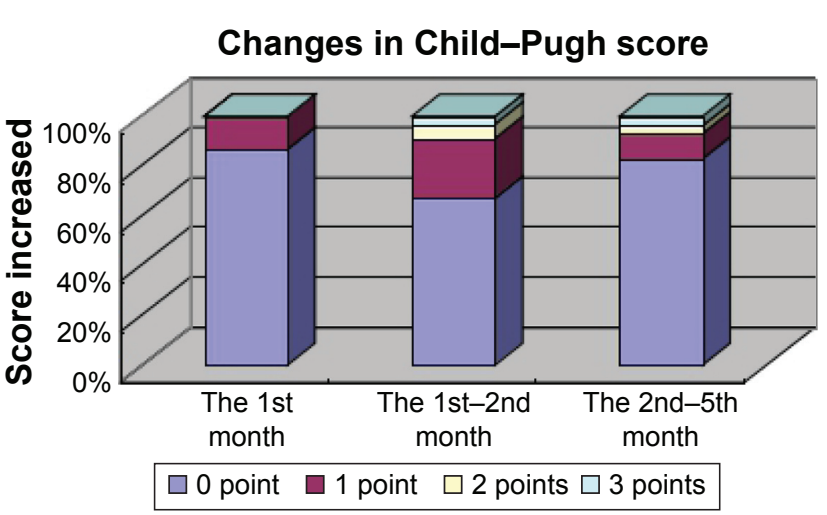

Figure I Changes of Child-Pugh score after CyberKnife stereotactic body radiation therapy.

$41(41 / 104,39.4 \%)$ patients and the increase was primarily found in the 3-10 week (about 1-2 months) period following CyberKnife SBRT. The majority (36/41, 87.8\%) experienced an increased CP score of one to two points, while five cases recorded an increased CP score of three points. RIHT, defined as an increase of at least two points within 3 months, occurred in 13 of the 104 patients $(13 / 104,12.5 \%)$.

During the 22-week follow-up period, 17 (16.3\%) patients indicated $\mathrm{CP}$ class progression. One $\mathrm{CP}$ class A patient and one $\mathrm{CP}$ class $\mathrm{B}$ patient experienced $\mathrm{CP}$ class progression to class $\mathrm{C}$ (Table 3 ).

\section{Parameters for predicting grade 2-3 hepatic toxicity}

We analyzed the clinical risk factors associated with grade 2-3 hepatic toxicity in HCC CyberKnife SBRT patients. The factors included gender, age, ECOG score, TNM staging, portal vein tumor thrombus, hepatitis B virus (HBV) infection, and the $\mathrm{CP}$ class recorded prior to CyberKnife radiotherapy. None of the clinical factors were found to be associated with grade $2-3$ hepatic toxicity $(P>0.05)$. The DVHsbased dosimetric parameters including GTV, CyberKnife SBRT fraction size, normal liver volume, $\mathrm{V}_{5}$ (defined as the percentage of normal liver volume receiving $>5 \mathrm{~Gy}$ ), $\mathrm{V}_{10}$, $\mathrm{V}_{15}, \mathrm{~V}_{20}, \mathrm{~V}_{25}$, and $\mathrm{V}_{30}$ were also analyzed. Using univariate

Table 3 Changes in Child-Pugh class following CyberKnife stereotactic body radiation therapy

\begin{tabular}{lllll}
\hline Time after & No of & \multicolumn{3}{l}{ CP class } \\
\cline { 3 - 5 } treatment (weeks) & patients & A & B & C \\
\hline Pretreatment (I-2) & 104 & 94 & 10 & 0 \\
Posttreatment (3-10) & 104 & 89 & 14 & I \\
Posttreatment (II-22) & 104 & 91 & II & 2 \\
\hline
\end{tabular}

Abbreviation: $\mathrm{CP}$, Child-Pugh.
Table 4 Multivariate analysis of significant variables in correlation with grade 2-3 hepatic toxicity

\begin{tabular}{lllllll}
\hline Variables & $\beta$ & SE & Wald & $P$-value & RR & 95\% Cl \\
\hline Fraction size & -0.077 & 0.206 & 0.140 & 0.708 & 0.926 & $0.618-1.387$ \\
GTV & 0.000 & 0.001 & 0.028 & 0.867 & 1.000 & $0.997-1.002$ \\
$\mathrm{~V}_{5}$ & -0.019 & 0.041 & 0.214 & 0.643 & $0.98 \mathrm{I}$ & $0.905-1.064$ \\
$\mathrm{~V}_{10}$ & -0.014 & 0.070 & 0.043 & 0.836 & 0.986 & $0.860-1.130$ \\
$\mathrm{~V}_{15}$ & -0.044 & 0.080 & 0.307 & 0.579 & 0.957 & $0.818-1.119$ \\
$\mathrm{~V}_{20}$ & -0.009 & 0.086 & $0.01 \mathrm{I}$ & 0.915 & 0.991 & $0.837-1.172$ \\
$\mathrm{~V}_{25}$ & 0.299 & 0.137 & 4.762 & 0.029 & 1.348 & $1.03 \mathrm{I}-1.764$ \\
$\mathrm{~V}_{30}$ & -0.173 & 0.105 & 2.716 & 0.099 & 0.842 & $0.685-1.033$ \\
\hline
\end{tabular}

Abbreviations: $\mathrm{Cl}$, confidence interval; GTV, gross tumor volume; RR, relative risk; SE, standard error.

analysis to evaluate the associations between the dosimetric parameters and grade 2-3 hepatic toxicity, we determined that GTV, CyberKnife SBRT fraction size, $\mathrm{V}_{5}, \mathrm{~V}_{10}, \mathrm{~V}_{15}$, $\mathrm{V}_{20}, \mathrm{~V}_{25}$, and $\mathrm{V}_{30}$ were associated with grade 2-3 hepatic toxicity $(P=0.016,0.029,0.041,0.021,0.007,0.002,0.001$, and 0.002 , respectively). Multivariate logistic regression analysis revealed that only $\mathrm{V}_{25}$ was a significant parameter associated with grade $2-3$ hepatic toxicity ( $P=0.029,95 \%$ CI 1.031-1.764; Table 4). As indicated in Figure 2, the area under the ROC curve (AUC) was 0.775 for $\mathrm{V}_{25}$, indicating that $\mathrm{V}_{25}$ with a cutoff value of $31.5 \%$ was adequate in predicting liver toxicity (grade 2-3). Liver toxicity (grade 2-3) was observed in 10 of 72 patients (13.9\%) with a $\mathrm{V}_{25}$ of $\leq 31.5 \%$ and in 14 of 32 patients (43.8\%) with a $\mathrm{V}_{25}$ of $>31.5 \%$. For the dosimetric parameter $\mathrm{V}_{25}$ with a cutoff value of $31.5 \%$, the sensitivity was 0.655 with a specificity of 0.827 .

\section{Parameters for predicting RIHT}

We analyzed the clinical risk factors of RIHT associated with CyberKnife SBRT. The factors analyzed included

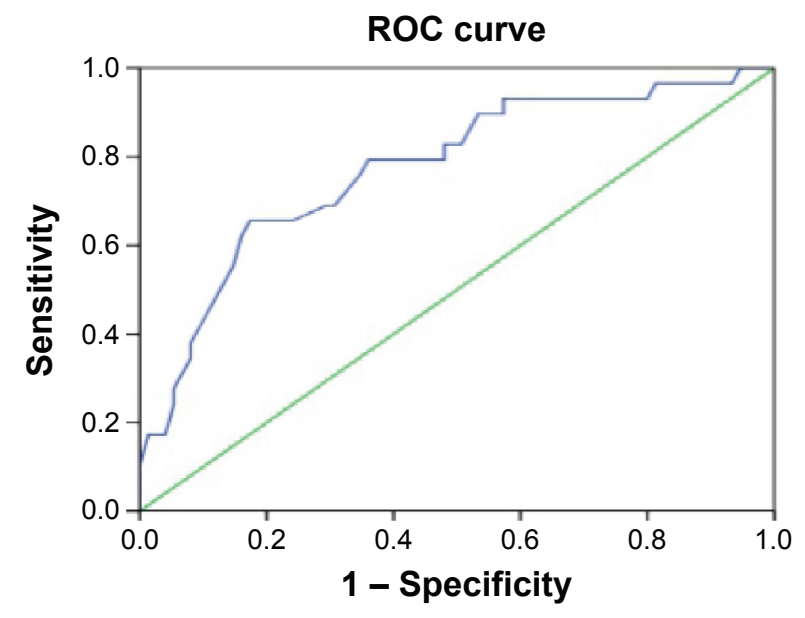

Figure $2 \mathrm{ROC}$ curve for $\mathrm{V}_{25}$.

Abbreviation: ROC, receiver operating characteristic. 


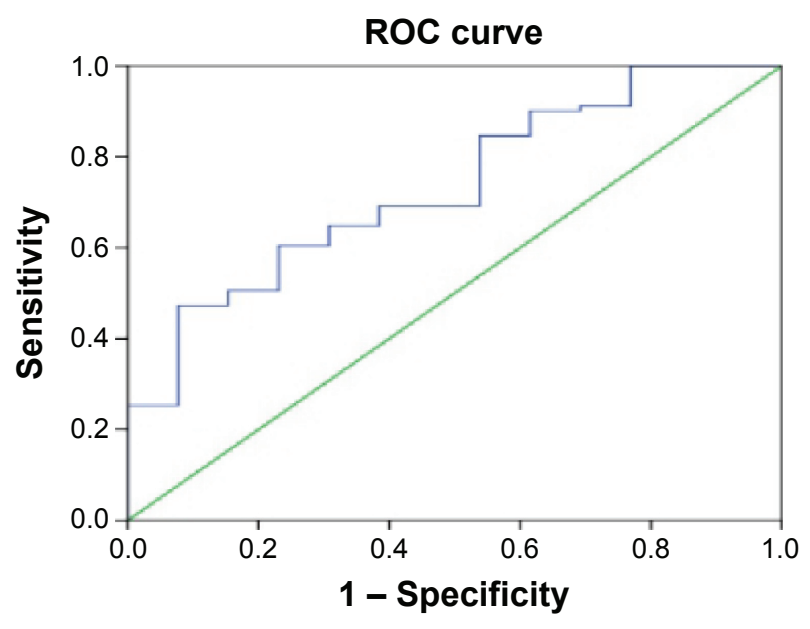

Figure 3 ROC curve for normal liver volume.

Abbreviation: ROC, receiver operating characteristic.

gender, age, ECOG score, TNM staging, portal vein tumor thrombus, $\mathrm{HBV}$ infection, and the $\mathrm{CP}$ class recorded prior to CyberKnife SBRT. None of the clinical factors were associated with RIHT $(P>0.05)$.

The DVHs-based dosimetric parameters analyzed included GTV, CyberKnife SBRT fraction size, normal liver volume, $\mathrm{V}_{5}, \mathrm{~V}_{10}, \mathrm{~V}_{15}, \mathrm{~V}_{20}, \mathrm{~V}_{25}$, and $\mathrm{V}_{30}$. Using univariate analysis to evaluate the associations between the DVHs-based dosimetric parameters and RIHT, we determined that only normal liver volume was found to be associated with RIHT $(P=0.008,<0.05)$. As shown in Figure 3, the AUC was 0.733 for normal liver volume, indicating that normal liver volume with the cutoff value of $1,094 \mathrm{~cm}^{3}$ was an appropriate predictor of RIHT. RIHT was observed in 4 out of 63 patients $(6.3 \%)$ with a normal liver volume $>1,094 \mathrm{~cm}^{3}$, and in 9 out of 41 patients $(22.0 \%)$ with a normal liver volume $\leq 1,094 \mathrm{~cm}^{3}$. For a normal liver volume with the cutoff value of $1,094 \mathrm{~cm}^{3}$, the sensitivity was 0.648 with a specificity of 0.692 .

\section{Discussion}

Dose escalation appears to be very important for the local control rates of radiotherapy in HCC patients, but the liver injury sustained by conventional radiotherapy has limited its application. HCC radiation therapy requires normal liver tissue-sparing radiation techniques, as there is a significant correlation between normal liver tissue threshold dose and the volume irradiated. ${ }^{13-15,18}$ The incidence of RIHT or radiation-induced liver disease (RILD) becomes significantly higher when the mean hepatic dose is $\geq 23$ Gy. ${ }^{13}$ CyberKnife SBRT is delivered in the setting of near real-time tracking of implanted fiducial markers, combined with respiratory motion modeling, to achieve submillimeter accuracy through the continual detection and correction of tumor motion throughout treatment. CyberKnife SBRT also allows the precise delivery of a large ablative radiation dose to the tumor while sparing the normal surrounding tissue, with less damage to healthy liver tissues in one to five fractions. During the past decade, a number of reports have documented the effect of SBRT on HCC as an alternative HCC treatment. The current consensus is that high-dose local radiation therapy alone or in combination with TACE could achieve a high rate of local control. ${ }^{19}$ CyberKnife SBRT greatly increases target dosage, thereby significantly improving the therapeutic efficacy of $\mathrm{HCC}$ radiation therapy.

In 2010, Kwon et al analyzed the long-term efficacy of SBRT for HCC on 47 patients who were ineligible for surgical resection or local ablation therapy. ${ }^{12}$ The median total dose was 33 Gy (range 30-39 Gy), delivered daily in three fractions. Their results revealed CR in 59.6\% and PR in $26.2 \%$ of the patients, and the overall 1- and 3-year survival rates were $92.9 \%$ and $58.6 \%$, respectively. Only one patient was found to have grade 4 liver toxicity. ${ }^{12}$ Their study indicated that CyberKnife SBRT was a promising noninvasive treatment for small $\mathrm{HCC}$ ineligible for local treatment or surgical resection. In 2011, Andolino et al evaluated the safety and efficacy of SBRT for the treatment of HCC in 60 patients, including 36 patients of CP class A and 24 of CP class B. ${ }^{10}$ Three months posttreatment, there were no $\geq$ grade 3 non-hematologic toxicities, though $13 \%$ of patients experienced an increase in hematologic and hepatic injury greater than grade and $20 \%$ experienced progression in $\mathrm{CP}$ class within three months of treatment. Our study revealed that the response rate $(\mathrm{CR}+\mathrm{PR})$ of $\mathrm{HCC}$ patients treated with CyberKnife SBRT was $80.8 \%$, the median survival time was 19 months, and the 1- and 2-year survival rates were $62 \%$ and $44 \%$, respectively. A possible reason for the lower survival rates in our study compared with the above-mentioned studies is that most of our patients were in an advanced stage ( $87.5 \%$ of stages III-IV patients).

In order to evaluate the effect of hepatic toxicity on the HCC patients treated with CyberKnife SBRT, we recruited 104 participants and measured the biochemical parameters and blood coagulation function during the treatment period. In the study group of $104 \mathrm{HCC}$ patients, 24 patients (23.1\%) experienced CTCAE V. 4.0 grade 2-3 hepatic toxicity, while no grade 4 hepatic toxicity was observed. The $\mathrm{CP}$ score increased significantly at 3-10 weeks and 11-22 weeks following treatment $(P<0.05)$ in $41(39.4 \%)$ patients, and the increase was predominantly detected about 1-2 months following CyberKnife SBRT. The majority of the patients 
(36/41, 87.8\%) experienced an increased CP score of one to two points, and five cases recorded an increased CP score of three points. RIHT is defined as an increase of at least two points within three months, and occurred in 13 of the 104 patients (12.5\%). During the 22-week follow-up period, $17(16.3 \%)$ patients indicated CP class progression, and $1 \mathrm{CP}$ class A patient and $1 \mathrm{CP}$ class B patient experienced CP class progression to class C. However, CyberKnife SBRT greatly reduced the irradiation doses delivered to the normal liver tissues, thus reducing the incidence of hepatic toxicity. The treatment was feasible in treating $\mathrm{HCC}$, and well tolerated with regard to slight hepatic toxicity.

Hepatic toxicity following radiotherapy is possibly related to tumor stage, CP class, liver function, GTV, total dose, or mean hepatic dose. ${ }^{13,20} \mathrm{We}$ analyzed the relationship between clinical factors and grade 2-3 hepatic toxicity. No parameters were found to be associated with grade 2-3 hepatic toxicity. RIHT occurred in 13 of the 104 patients (12.5\%), and none of the factors included in the CP class prior to CyberKnife radiotherapy and GTV were associated with RIHT $(P>0.05)$. Ten HCC patients with CP class B liver cirrhosis were included in the study. We used univariate analysis to evaluate RIHT and only normal liver volume was associated with RIHT $(P>0.05)$. Therefore, CyberKnife SBRT may be an effective therapy with a good hepatic toxicity profile, especially in HCC patients with CP class B liver cirrhosis. Previous research regarding the dosimetric parameters predicting hepatic toxicity was based on the data from conformal radiotherapy or IMRT. The planning and delivery method of CyberKnife SBRT differs from that of conformal radiotherapy or IMRT. A larger sample is needed to validate the safety of hepatic toxicity.

Our previous study has demonstrated that $\mathrm{V}_{20}$ is a unique significant dosimetric predictor for RILD risks in primary liver carcinoma patients with CP Grade A cirrhosis treated with hypofractionated conformal radiotherapy. ${ }^{21}$ Therefore, we analyzed the relationship between the DVHs parameters and hepatic toxicity in HCC patients treated with CyberKnife SBRT. The ROC curve indicated in Figure 2 revealed that $\mathrm{V}_{25}$ with a cutoff value of $31.5 \%$ was an appropriate predictor of hepatic toxicity (grade 2-3), with a sensitivity of 0.655 and a specificity of 0.827 . Furthermore, $V_{25}$ was identified as the independent factor and could serve as an effective dosimetric parameter to assess the risk of hepatic toxicity. This is in line with the $\mathrm{V}_{20}$ reported by Liang et al who analyzed the results from $\mathrm{HCC}$ patients receiving hypofractionated conformal radiotherapy. ${ }^{21}$ In 2010 , Seok et al reported that total liver volume receiving $<18$ Gy should be $>800 \mathrm{~cm}^{3}$ to reduce the risk of deterioration of hepatic function. ${ }^{20}$ It also suggests that liver volume may be related to the occurrence of RIHT. In our evaluation of the associations between the DVHs-based dosimetric parameters and RIHT, we found that only the normal liver volume was associated with RIHT $(P=0.008)$. For normal liver volume with a cutoff value of $1,094 \mathrm{~cm}^{3}$, the sensitivity was 0.648 with a specificity of 0.692 . These data verified the well-known assumption that the tolerance of the liver to external beam irradiation depends on the volume of liver irradiated.

As a limited number of eligible patients were included in this study, the indications and contraindications of CyberKnife SBRT in HCC should be further investigated. The curative effects of CyberKnife SBRT should also be further studied in large prospective cohort clinical studies. Due to the slight hepatic toxicity of CyberKnife SBRT in $\mathrm{HCC}$, prospective studies of CyberKnife SBRT combined with other treatment modalities, including chemotherapy, may also be promising.

\section{Conclusion}

In conclusion, despite the unfavorable impact on liver functioning during the first month of treatment, our study suggests that CyberKnife SBRT is feasible and safe for treating HCC patients, including those with $\mathrm{CP}$ class $\mathrm{B}$ liver cirrhosis and is associated with a good tumor response rate. $\mathrm{V}_{25}$ and normal liver volume can serve as effective dosimetric parameters to assess the risk of grade 2-3 hepatic injury and RIHT, respectively.

\section{Acknowledgment}

This work was supported by the Education Department of Fujian Province to Hai-Jie Lu (JK2015019).

\section{Disclosure}

The authors report no conflicts of interest in this work.

\section{References}

1. Maluccio M, Covey A. Recent progress in understanding, diagnosing, and treating hepatocellular carcinoma. CA Cancer J Clin. 2012;62(6): 394-399.

2. Bosch FX, Ribes J, Diaz M, Cleries R. Primary liver cancer: worldwide incidence and trends. Gastroenterology. 2004;127(5 Suppl 1):S5-S16.

3. Siegel R, Naishadham D, Jemal A. Cancer statistics, 2012. CA Cancer J Clin. 2012;62(1):10-29.

4. Bruix J, Sherman M. Management of hepatocellular carcinoma: an update. Hepatology. 2011;53(3):1020-1022.

5. Shiina S, Teratani T, Obi S, Hamamura K, Koike Y, Omata M. Nonsurgical treatment of hepatocellular carcinoma: from percutaneous ethanol injection therapy and percutaneous microwave coagulation therapy to radiofrequency ablation. Oncology. 2002;62(Suppl 1):64-68. 
6. Jeroen D, Hannah VM, Vincent V, et al. Transcatheter arterial chemoembolization with doxorubicin-eluting superabsorbent polymer microspheres in the treatment of hepatocellular carcinoma: midterm follow-up. J Vasc Interv Radiol. 2014;25(2):248-255.

7. Keating G, Santoro A. Sorafenib: a review of its use in advanced hepatocellular carcinoma. Drugs. 2009;69(2):223-240.

8. Sarfaraz M. CyberKnife ${ }^{\circledR}$ robotic arm stereotactic radiosurgery. J Am Coll Radiol. 2007;4(8):563-565.

9. Coste-Manière E, Olender D, Kilby W, Schulz RA. Robotic whole body stereotactic radiosurgery: clinical advantages of the CyberKnife integrated system. Int J Med Robot. 2005;1(2):28-39.

10. Andolino DL, Johnson CS, Maluccio M, et al. Stereotactic body radiotherapy for primary hepatocellular carcinoma. Int J Radiat Oncol Biol Phys. 2011;81(4):e447-e453.

11. Sanuki N, Takeda A, Kunieda E. Role of stereotactic body radiation therapy for hepatocellular carcinoma. World J Gastroenterol. 2014; 20(12):3100-3111.

12. Kwon JH, Bae SH, Kim JY, et al. Long-term effect of stereotactic body radiation therapy for primary hepatocellular carcinoma ineligible for local ablation therapy or surgical resection. Stereotactic radiotherapy for liver cancer. BMC Cancer. 2010;10:1-10.

13. Liang SX, Zhu XD, Xu ZY, et al. Radiation-induced liver disease in three-dimensional conformal radiation therapy for primary liver carcinoma: the risk factors and hepatic radiation tolerance. Int J Radiat Oncol Biol Phys. 2006;65(2):426-434.

14. Cheng JC, Wu JK, Huang CM, et al. Radiation-induced liver disease after three-dimensional conformal radiotherapy for patients with hepatocellular carcinoma: dosimetric analysis and implication. Int J Radiat Oncol Biol Phys. 2002;54(1):156-162.
15. Lawrence TS, Robertson JM, Anscher MS, Jirtle RL, Ensminger WD, Fajardo LF. Hepatic toxicity resulting from cancer treatment. Int J Radiat Oncol Biol Phys. 1995;31(5):1237-1248.

16. Ye SL. [Expert consensus on standardization of the management of primary liver cancer]. Zhonghua Gan Zang Bing Za Zhi. 2009;17(6): 403-410. Chinese.

17. Liaw YF, Sung JJ, Chow WC, et al. Lamivudine for patients with chronic hepatitis B and advanced liver disease. N Engl J Med. 2005;351(15): 1521-1531.

18. Park HC, Seong J, Han KH, Chon CY, Moon YM, Suh CO. Dose response relationship in local radiotherapy for hepatocellular carcinoma. Int J Radiat Oncol Biol Phys. 2002;54(1):150-155.

19. Kang JK, Kim MS, Cho CK, et al. Stereotactic body radiation therapy for inoperable hepatocellular carcinoma as a local salvage therapy after incomplete transarterial chemoembolization. Cancer. 2012;118(21): 5424-5431.

20. Seok HS, Byung OC, Ryeong RM, et al. Stereotactic body radiotherapy for patients with unresectable primary hepatocellular carcinoma: dosevolumetric parameters predicting the hepatic complication. Int J Radiat Oncol Biol Phys. 2010;78(4):1073-1080.

21. Liang SX, Huang XB, Zhu XD, et al. Dosimetric predictor identification for radiation-induced liver disease after hypofractionated conformal radiotherapy for primary liver carcinoma patients with Child-Pugh Grade A cirrhosis. Radiother Oncol. 2011;98(2):265-269.
OncoTargets and Therapy

\section{Publish your work in this journal}

OncoTargets and Therapy is an international, peer-reviewed, open access journal focusing on the pathological basis of all cancers, potential targets for therapy and treatment protocols employed to improve the management of cancer patients. The journal also focuses on the impact of management programs and new therapeutic agents and protocols on

\section{Dovepress}

patient perspectives such as quality of life, adherence and satisfaction. The manuscript management system is completely online and includes a very quick and fair peer-review system, which is all easy to use. Visit http://www.dovepress.com/testimonials.php to read real quotes from published authors. 